\title{
System Availability Modelling and Optimization considering Multigeneral Quality Characteristics
}

\author{
Junliang Li $\mathbb{D},,^{1,2}$ Yueliang Chen $\mathbb{D}^{1},{ }^{1}$ and Yong Zhang $\mathbb{D}^{1}$ \\ ${ }^{1}$ Qingdao Campus of Naval Aeronautics University, Qingdao 266041, China \\ ${ }^{2}$ 92635th Uint of PLA, Qingdao 266041, China \\ Correspondence should be addressed to Yueliang Chen; cyl0532@sina.com
}

Received 22 September 2019; Revised 22 November 2019; Accepted 7 January 2020; Published 20 February 2020

Academic Editor: Konstantina Skouri

Copyright (c) 2020 Junliang Li et al. This is an open access article distributed under the Creative Commons Attribution License, which permits unrestricted use, distribution, and reproduction in any medium, provided the original work is properly cited.

This paper analyzes the necessity that multigeneral quality characteristic parameters should be considered in availability modelling and optimization and then constructs an availability model including the indicators of reliability, maintainability, supportability, testability, and environmental factor. In the research process, it is assumed that the system has soft and hard failure mode, adopting mixed maintenance strategies, system delay time, and repair time, and test time are independent. On the basis of this, the single parameter, binary parameters, and multiparameters optimization were carried out with the maximum availability as the optimization function. Especially, the multiparameters availability optimization model in time dimension was designed based on particle swarm optimization. The design method was proved feasible through a numerical example, which can provide method support for the design and evaluation of general quality characteristic thresholds in the life cycle of a repairable system.

\section{Introduction}

With the progress of science and technology, the equipment configuration is becoming more and more complex and the function of general quality characteristics such as reliability and maintainability is becoming more and more important. However, the traditional design method does not well accommodate the design requirement for multiple general quality characteristics of complex systems, which increases life-cycle costs and decreases support effectiveness. So, the individual design for reliability, maintainability, and supportability (RMS) should be improved into the integrated design. Availability is the probability that the system is capable of conducting its required function when it is called upon given that it is not failed or undergoing a repair action [1-4]. The availability is not only a function of reliability, but it is also a function of maintainability and supportability.

Barlow [1] analyzed the system availability using the Markov process and also discussed the concepts of mathematical theory of reliability, when system failure and repair time obey exponential distributions. From then, more researchers are focused on the system instantaneous availability modelling, but the steady-state availability is most important in the engineering practice, which was an important indicator to balance costs and benefits, such as military and space systems [5-7].

Sarkar and Sarkar [8-10] studied the instantaneous availability and steady-state availability for an inspectionbased system using a recursive method, with lifetime and repair time following discrete distribution. Cui and Xie [11] assumed that the periodic inspections take place at fixed time points after repairs or replacements in case of failures. Some general results on the instantaneous availability and the steady-state availability were presented under the assumption of random repair or replacement time. Furthermore, $\mathrm{Li}$ et al. $[12,13]$ analyzed the availability for a periodical inspection system with arbitrary lifetime and repair time distribution under a perfect repair policy, and the relationship between the inspection period and availability was also analyzed. It should be noted that all the abovementioned results were obtained under the assumption that down time caused by preventive maintenance (PM) time is negligible. Tang et al. [14] investigated the availability of a periodically inspected system, considering nonnegligible down time 
caused by inspection, and constructed an analytical model. Ebeling [2] proposed that the steady-state availability depends on the system Mean Time Between Failure (MTBF) and Mean Time To Repair (MTTR), as well as on the maintenance and supply delay time, where MTBF is the important indicator of system reliability, MTTR is the important indicator of system maintainability, and the maintenance and supply delay time reflect the ability of the support system. Gao et al. [15] analyzed the operational availability and brought logistic delay probability into the expression of availability. Ding [16] presented three kinds of practical theoretical models for the toplevel design for RMS, considering the system configuration, MTBF and MTTR, as well as mean delay time. However, there is no literature which discussed the testability and environmental adaptability factors in the availability model.

The optimization of availability, system costs, and risk is another important problem in reliability engineering. These research studies focus on the measurement and trade-offs between one parameter or multiple parameters between availability, reliability, and economic cost [17-20].

The stochastic process theory based on the Markov process is the main method to solve this problem [18], which provides various possibilities and combinations of probabilities associated with the different states. Qiu and Cui $[21,22]$ discussed the optimal model for a system with competing failure or multiple failure, and the result can be utilized to obtain the optimal inspection interval that maximizes the system steady-state availability or minimizes the average long-run cost rate. $\mathrm{Li}$ et al. [23] investigated optimal maintenance policies for multicomponent systems under periodic and opportunistic inspections with multiple control limits, in which the wait time is considered.

In general, a nonperiodic condition-based PM policy with different condition variables is often more effective than a periodic age-based policy for deteriorating complex repairable systems. Since the increase in service time and changes in the service environment will affect the intrinsic reliability of the system, Lin et al. [24] developed an optimal model, in which system reliability is estimated and used as the condition variable, the system availability and cost are the optimal object, and three reliability-based PM models are then developed with consideration of different scenarios which can assist in evaluating the maintenance cost for each scenario. Hajipour and Taghipour [25] proposed a model to find the optimal nonperiodic inspection interval over a finite planning horizon for two types of multicomponent repairable systems with hard-type and soft-type components and then integrated the simulation model with a genetic algorithm to achieve the optimal scheme. All these papers $[24,25]$ assumed that reliability varies over different PM periods and that the change is primarily generated by PM behavior, and the PM interval is determined based on the same reliability threshold and maintenance cost. They introduced the age reduction factor, but they did not consider the impact of the environment factors on the intrinsic reliability of the system.
Although many outstanding scholars have made extensive research on availability, modelling, and optimization, there are still some drawbacks:

(1) For different research objects, perfect maintenance or imperfect maintenance is only considered individually instead of simultaneously in one availability model.

(2) There is no availability model that comprehensively considers reliability, maintainability, support-ability, test-ability, and environmental adaptability.

(3) There is no comprehensive optimization model considering the multigeneral quality characteristics.

Our study has made two main contributions. Firstly, we propose the instantaneous availability and steady-state availability of a periodically inspected system with multiple quality factors, which include the indicators of reliability, maintainability, supportability, testability, and environmental adaptability; secondly, the availability optimization model with multiparameters based on particle swarm optimization was designed in time dimension. The application of the results in this paper is illustrated through a numerical example.

The remainder of this paper is structured as follows: Section 2 describes the system with multiple assumptions. In Section 3, the steady-state availability with multiple factors and particle swarm optimization algorithm are analyzed. In Section 4, we demonstrate the developed model and the optimal method through a numerical example. In Section 5, conclusion and future research works are given.

\section{System Description}

The specific assumptions used for availability and optimal modelling are summarized as follows.

2.1. Assumption 1: The System Failure Modes. Assume that the system has hard failures and soft failures; the former directly brings the system down, while the latter can only be found during PM intervals. After a hard fault occurs, the system needs to be shut down for corrective maintenance and a perfect maintenance strategy will be adopted.

2.2. Assumption 2: The Corrective Maintenance (CM) Time and the Preventive Maintenance (PM) Time. The corrective maintenance time is represented by the random variable $Y_{1}$, which consists of delay time $Z$ and repair time $T_{c}$.

$$
Y_{1}=T_{c}+Z \text {, }
$$

where the variables $T_{c}$ and $Z$ are independent of each other and $T_{c}$ is the repair and/or replacement time. $Z$ is delay time, since the fails are triggered unexpectedly. In engineering practice, such as aircraft and helicopter, spares delay is the main factor causing delay, so the system delay time can be defined as

$$
Z=(1+\zeta) Z_{1}
$$


where $Z$ is the system delay time, $Z_{1}$ is the spare delay time, and $\zeta$ is the decision variable, with $0 \leq \zeta<1$, which is caused by other things except spare.

PM is performed at fixed intervals $T$ after the former PM activity, and after the inspection, necessary activities are taken to restore the system by imperfect maintenance. Such imperfect maintenance activities can make the effective age of the system younger and result in an improved operating condition. Especially, after each PM activities, the systems is available.

The preventive maintenance time is represented by the random variable $Y_{2}$, which consists of preventive repair time $T_{p}$ and inspection/test time $T_{\text {ins }}$.

$$
Y_{2}=T_{\text {ins }}+T_{p} .
$$

The variables $T_{\text {ins }}$ and $T_{p}$ are independent of each other and $T_{\text {ins }}$ is the test time, which includes the time of diagnostic instruments setting and is also referred to as fault isolation time.

2.3. Assumption 3: Environmental Adaptability Factor. An environmental factor is a parameter that characterizes how fast the same product fails in different environments. It reflects the level of environmental impact on product reliability. Before we study environmental factors, there are two basic assumptions.

Firstly, we assume that the system failure mechanism is unchanged under changed environment.

Secondly, we assume that the system life distribution belongs to the same distribution type, and the relevant parameters are different under changed environment.

In the actual working environment, the system may be affected by a variety of environmental stresses (corrosion, vibration, temperature, etc.), which can be written as $\mathbf{m}=\left\{x 1, x 2, \ldots, x_{n}\right\}$. It is difficult to accurately express it with an analytical model. Here, we apply the model in Yang et al. [26], which assumed four factors (corrosion, vibration, temperature, and humidity) frequently affecting system intrinsic reliability in a linear dynamical system.

$$
\begin{aligned}
& \lambda^{(1)}(t)=\lambda(t), \\
& \lambda^{(2)}(t)=I_{2} \lambda\left(t^{\prime}+T_{1}-\xi_{1} T_{1}\right), \\
& \lambda^{(3)}(t)=I_{3} \lambda_{2}\left(t^{\prime}+T_{2}-\xi_{2} T_{2}\right), \\
& \quad \vdots \\
& \lambda^{(i)}(t)=I_{i} \lambda\left(t^{\prime}+T_{i-1}-\xi_{i-1} T_{i-1}\right), \quad i=1,2, \ldots, N,
\end{aligned}
$$

where $\xi_{i}$ represents the effective age of the system immediately after the $i^{\text {th }} \mathrm{PM}$ activity, $0 \leq \xi_{i} \leq 1$, and $\lambda(t)$ is the system extrinsic failure rate; $I_{i}=\sum_{k=1}^{n} p_{k} x_{k}$ denotes the degree of the operating environment effects on the intrinsic reliability subjected to factor $x_{k}, p_{i}$ is the weight of factor $i$, which is set between 0 and 1 , and $\sum_{k=1}^{n} p_{k}=1$.

\section{System Availability Analysis}

3.1. System Steady-State Availability. Assume that the system consists of alternating processes of work and maintenance, as shown in Figure 1.

It is assumed that $X$ is the system life which obeys the general distribution $F(t)$. When the system works to the specified PM time without failure, the preventive maintenance will be adopted, as shown in Figure 1(A). When the system fails within the PM interval, the system performs corrective maintenance and takes the perfect maintenance strategies, as shown in Figure 1(B).

Let assume that $X$ is the life:

$$
\begin{gathered}
\tilde{X}= \begin{cases}X, & \text { when } X \leq T, \\
T, & \text { when } X>T,\end{cases} \\
\tilde{Y}= \begin{cases}Y_{1}, & \text { when } X \leq T, \\
Y_{2}, & \text { when } X>T .\end{cases}
\end{gathered}
$$

When the system is available at time 0 , the system instantaneous availability at time $t$ can be gained [27].

$$
A(t)=P\{\tilde{X}>t\}+P\{\tilde{X}+\widetilde{Y} \leq t\} * A(t) .
$$

Then, make Laplace transform for equation (7):

$$
A(s)=\frac{\int_{0}^{\infty} e^{-s t} P\{\widetilde{X}>t\} \mathrm{d} t}{1-\int_{0}^{\infty} e^{-s t} d P\{\widetilde{X}+\widetilde{Y} \leq t\} \mathrm{d} t} .
$$

Since

$$
P\{\tilde{X}>t\}= \begin{cases}P\{X>t\}, & \text { when } t \leq T \\ 0, & \text { when } t>T\end{cases}
$$

So,

$$
\begin{aligned}
\int_{0}^{\infty} e^{-s t} P\{\tilde{X}>t\} \mathrm{d} t= & \int_{0}^{T} e^{-s t} \bar{F}(t) \mathrm{d} t, \\
\int_{0}^{\infty} e^{-s t} P\{\tilde{X}+\widetilde{Y} \leq t\}= & E\left\{e^{-s(\widetilde{X}+\widetilde{Y})}\right\}, \\
= & \int_{0}^{\infty} E\left\{e^{-s(\widetilde{X}+\widetilde{Y})} \mid X=t\right\} \mathrm{d} P\{X \leq t\}, \\
= & \int_{0}^{T} E\left\{e^{-s\left(t+Y_{1}\right)}\right\} \mathrm{d} F(t) \\
& +\int_{T}^{\infty} E\left\{e^{-s\left(T+Y_{2}\right)}\right\} \mathrm{d} F(t), \\
= & E\left\{e^{-s Y_{1}}\right\} \int_{0}^{T} e^{-s t} \mathrm{~d} F(t) \\
& +E\left\{e^{-s Y_{2}}\right\} e^{-s T} \mathrm{~d} F(t), \\
= & \widehat{G}_{1}(s) \int_{0}^{T} e^{-s t} \mathrm{~d} F(t)+\widehat{G}_{2}(s) e^{-s T} \mathrm{~d} F(t) .
\end{aligned}
$$




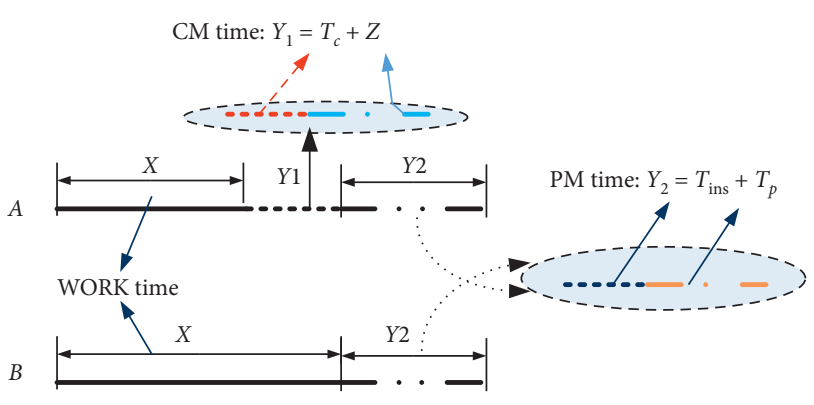

Figure 1: System operation state.

In the equation (11), $\widehat{G}_{1}(s)$ and $\widehat{G}_{2}(s)$ are the Laplace transform.

Form for $G_{1}(t)$ and $G_{2}(t)$.

$$
A^{*}(s)=\frac{\int_{0}^{T} e^{-s t} \bar{F}(t) \mathrm{d} t}{1-\widehat{G}_{1}(s) \int_{0}^{T} e^{-s t} \mathrm{~d} F(t)-\widehat{G}_{2}(s) e^{-s T} \bar{F}(T)} .
$$

When $F(t), G_{1}(t)$, and $G_{2}(t)$ are lattice distributions, $P\{\tilde{X}+\widetilde{Y} \leq t\}$ is also lattice distribution. According to Tobel theorem and l'Hôpital's rule, the steady-state availability is denoted as

$$
\begin{aligned}
A & =\lim _{t \rightarrow \infty} A(t)=\lim _{t \rightarrow \infty} \frac{1}{t} \int_{0}^{T} A(u) \mathrm{d} u=\lim _{s \longrightarrow 0} s A^{*}(s), \\
& =\frac{\int_{0}^{T} \bar{F}(t) \mathrm{d} t}{\int_{0}^{T} \bar{F}(t) \mathrm{d} t+E\left(Y_{1}\right) F(T)+E\left(Y_{2}\right) R(T)}
\end{aligned}
$$

So, the system steady-state availability is given by the first PM interval:

$$
A=\frac{\int_{0}^{T} R(t) \mathrm{d} t}{\int_{0}^{T} R(t) \mathrm{d} t+E\left(Y_{2}\right)+(1-R(T)) \cdot\left(E\left(T_{c}\right)+E[Z]\right)},
$$

where $E(Z)$ is the mean time of delay time; $E\left(Y_{2}\right)$ is the mean time of PM, which includes test time and repair and/or replacement time; $T$ is the fixed time, and let $E\left(Y_{2}\right)=\tau^{\prime}$. Therefore, system steady-state availability in the $k+1 \mathrm{PM}$ interval is given as

$$
\begin{aligned}
A & =\frac{E(X)}{\sum_{k=0}^{n}\left(E(X)+E\left(Y_{2}\right)+\left(1-R^{(k)}(T)\right) \cdot\left(E\left(T_{c}\right)+E(Z)\right)\right)}, \\
& =\frac{\sum_{k=0}^{n} \int_{k T}^{(k+1) T} R^{(k)}(t) \mathrm{d} t}{\sum_{k=0}^{n}\left(E(X)+E\left(Y_{2}\right)+\left(1-R^{(k)}(T)\right) \cdot\left(E\left(T_{c}\right)+E(Z)\right)\right)}, \\
& =\frac{\sum_{k=0}^{n} \int_{k T}^{(k+1) T} R^{(k)}(t) \mathrm{d} t}{\sum_{k=0}^{n}\left(E(X)+E\left(Y_{2}\right)+\left(1-R^{(k)}(T)\right) \cdot\left(E\left(T_{c}\right)+E(Z)\right)\right)}
\end{aligned}
$$

where $R^{(k)}(t)$ is the reliability in the $k^{\text {th }}$ stage, which indicates the system reliability after $k-1^{\text {th }} \mathrm{PM}$ activity, and the environmental adaptability factor is $I_{k}$. Hence, the reliability

$$
R^{(k)}(t)=I_{k} R^{(k-1)}(t)
$$

$R^{(i)}(t)$ is decided by the intrinsic reliability and age reduction factor, as well as environmental adaptability factor.

Obviously, from equation (14) to equation (16), we can see the parameters of reliability, maintainability, supportability, testability, and environmental are in the new mathematical model.

PM interval is an important characterization parameter for the reliability threshold, the mean delay time is an important characteristic parameter of the supportability, and PM time and CM time are characterization parameters of maintainability.

The model for steady-state availability is also given by Chinese Military Standards (GJB) 1909A-2009 [28], which is

$$
A=\frac{E(X)}{E(X)+T_{c}+T_{p}+E(Z)} \text {. }
$$

Through comparison of equation (15) with equation (17), it can be seen that the model in the paper considers more factors and more effectively reflects the availability of the system under real conditions.

3.2. Optimization Algorithm Based on PSO. In order to consider various possible combinations of random failure and repair rate to achieve optimal availability of a system, PSO is applied for predicting the availability in process industry [29-31].

Let $X_{i}(t)=\left\{x_{i, 1}(t), \ldots, x_{i, n}(t)\right\}$ and $V_{i}(t)=\left\{v_{i, 1}(t), \ldots\right.$, $\left.v_{i, n}(t)\right\}$, respectively, are the position and the velocity of particle $i$ in time $t$, in the $n$-dimensional search space. Considering that $p$ Best $_{i}(t)=\left\{p\right.$ Best $_{i, 1}(t), \ldots$, Best $\left._{i, n}(t)\right\}$ is the best position already found by particle $i$ until time $t$ and $g$ Best $_{i}(t)=\left\{g\right.$ Best $\left._{i, 1}(t), \ldots, g_{\text {Best }}{ }_{i, n}(t)\right\}$ is the best position already found by a neighbor until $t$, the PSO updating rules for velocity and position are given by

$$
\left\{\begin{array}{l}
v_{i, j}(t+1)=w v_{i, j}(t)+c_{1} r_{1}\left[p_{i, j}-x_{i, j}(t)\right]+c_{2} r_{2}\left[p_{g, j}-x_{i, j}(t)\right], \\
x_{i, j}(t+1)=x_{i, j}(t)+v_{i, j}(t+1), \quad j=1,2, \ldots, d,
\end{array}\right.
$$

where $r_{1}$ and $r_{2}$ are random numbers between 0 and 1 , coefficients $c_{1}$ and $c_{2}$ are given acceleration constants towards pBest and $g$ Best, respectively, and $w$ is the inertia weight. The inertia weight $w$ is the responsible for the scope of the exploration of the search space. High values of $w$ may promote global search and improves the quality of solutions, while low values lead to local search. A common approach to provide balance between global and local search is to linearly decrease $w$ during the search process. In this paper, we utilize the adaptive weights policy, which can constantly adjust the weights during the calculation process and obtain the global optimal value. 


$$
w= \begin{cases}w_{\min }-\frac{\left(w_{\max }-w_{\min }\right) \cdot\left(f-f_{\min }\right)}{\left(f_{\mathrm{avg}}-f_{\min }\right)}, & f \leq f_{\mathrm{avg}}, \\ w_{\max }, & f>f_{\text {avg }},\end{cases}
$$

where $w_{\max }$ and $w_{\min }$ are, respectively, the max and $\min w . f$ is the objective function, $f_{\text {avg }}$ and $f_{\min }$ are, respectively, the average and $\min f$. Also, the system optimal model can be seen in Figure 2.

In Figure 2, $D$ is the particle dimension, $N$ is the particle number, $M$ is the max iterative number, and the objective function is max availability.

\section{Numerical Example}

4.1. System Analysis. In this section, a numerical example to demonstrate the correctness and feasibility of the proposed models and algorithm is provided.

Assume that there are two failure modes in the system. The first one is a hard fault. If it occurs, the system will be down, and the maitenance should be taken with a perfect maintenance strategy; the second is the soft fault because the soft fault does not affect the normal operation of the system. If the occurrence can be repaired within the PM interval, an imperfect maintenance strategy is adopted; therefore, when calculating the system mission reliability, only the hard failure model is considered.

Assume that the system hard fault obeys the Weibull distribution, and suppose that the parameters of the intensity function are estimated as $\theta=1000$ hour and $\beta=2$. By giving the hazard rate, the system reliability and hazard rate of hard fault are, respectively,

$$
\begin{aligned}
& R(t)=e^{-(t / \theta)^{\beta}}, \\
& \lambda(t)=\frac{\beta}{\theta}\left(\frac{t}{\theta}\right)^{\beta-1}, \quad \theta>0, \beta>0, t>0 .
\end{aligned}
$$

When the reliability threshold $R_{1, c}=0.9$, system lifetime is $x \approx 320$ hour. Also, the soft fault obeys the exponential distribution, $R_{2}(t)=e^{-\lambda t}=e^{-0.0002 t}$, if $R_{2, C}=0.9, x \approx$ 525 hour.

According to the basic principle of competition failure, the Weibull distribution should also be selected as the system reliability model (Figure 3). Since preventive maintenance takes an imperfect maintenance strategy, it is assumed here that the age reduction factor is given as equation (22), which is marginally increasing with the frequency of PM [24].

$$
\xi_{i}=\frac{i}{2 i+1} \text {. }
$$

Then, the system hazard rate is in the $i^{\text {th }}$ interval:

$$
\lambda_{i}(t)=\lambda\left(t^{\prime}+\frac{i^{2}}{2 i-1} T\right), \quad t^{\prime} \in[(i-1) T, i T] .
$$

Also, the system reliability

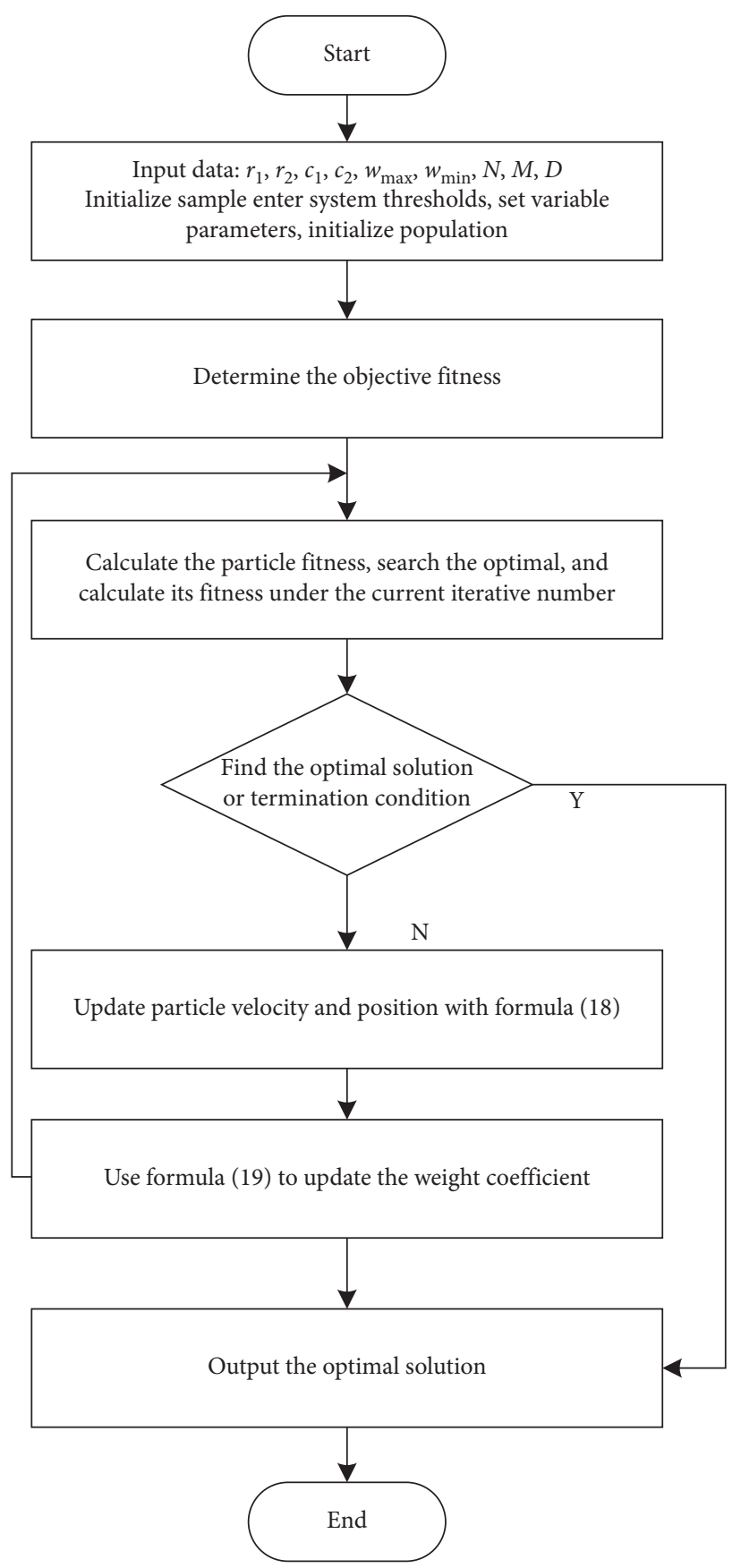

FIGURE 2: Flowchart of improved particle swarm optimization algorithm.

$$
R^{(i)}(t)=m_{k} e^{-\int_{0}^{T} \lambda\left(u+\left(i^{2} / 2 i-1\right) T\right) \mathrm{d} u} .
$$

Then, the up time for the system in the first interval is given as

$$
E(X)=\int_{0}^{T} R(t) \mathrm{d} t=\int_{0}^{T} e^{-(t / \theta)^{\beta}} \mathrm{d} t .
$$

Also, the CM time $E\left(Y_{1}\right)=a+(1+\zeta) b, a$ and $b$ are constant, and the PM time $E\left(Y_{2}\right)=\tau^{\prime}$. 
Then, the system steady-state availability in the first stage is given by

$$
A=\frac{\int_{0}^{T} R(t) \mathrm{d} t}{\int_{0}^{T} R(t) \mathrm{d} t+\tau^{\prime}+(1-R(T) \cdot(a+(1+\zeta)) b)}
$$

Equation (26) shows the analytic model of availability, and if we know the values of $a$ and $b$, we can utilize equations (14) and (15) to compute the system steady-state availability in the life cycle.

$$
A=\frac{500 \cdot \pi^{1 / 2} \cdot \operatorname{erf}[T / 1000]}{500 \cdot \pi^{1 / 2} \cdot \operatorname{erf}[T / 1000]+\tau^{\prime}+\left[1-\exp \left(-(T / 1000)^{2}\right)\right] \cdot[a+(1+\zeta) \cdot b]}
$$

In the next section, we will take the maximum availability as the objective function to optimize the problem under three assumptions, respectively.

4.2. Single Parameter Optimization. Preventive maintenance period is a major factor influencing the availability of repairable equipment [21-23], since frequent PM activities often result in high maintenance costs and poor system availability, while insufficient PM activities may not accomplish the goal of quality assurance. In this part, we suppose all parameters in equation (15) are known, except the PM interval. We consider the steady-state availability as a function of inspection interval, and we assume the maintenance interval T as a variable $\tau$. Let $\theta=1000, \beta=2, \tau^{\prime}=2$, $a=4, b=2, \zeta=0.05$, and $I_{i} \equiv 1$; then, substituting these parameters into equation (27), we can obtain the system availability function:

$$
A=\frac{500 \cdot \pi^{1 / 2} \cdot \operatorname{erf}[\tau / 1000]}{500 \cdot \pi^{1 / 2} \cdot \operatorname{erf}[\tau / 1000]+2+\left[1-\exp \left(-(\tau / 1000)^{2}\right)\right] \cdot[4+(1+\zeta) \cdot 2]}
$$

Then, utilize the MATLAB2016A software to calculate the system steady-state availability, as shown in Figure 4.

As shown in Figure 3, the system availability increases monotonically with operation hours because the system failure rate is low. If preventive maintenance is overtaken, it will cause the system to have long down time and low availability. When all the parameters are set, the availability is 0.99 and the PM interval is 235 operation hours.
4.3. Binary Parameter Optimization. In this part, we suppose all parameters in equation (16) are known, except the PM interval and $\tau^{\prime}$. We consider the steady-state availability as a function of inspection interval and $\tau^{\prime}$. Let $\theta=1000, \beta=2$, $a=4, b=2$, and $\zeta=0.05$, and then substituting these parameters into equation (27), we can obtain the system availability function as shown in Figure 5.

$$
A=\frac{500 \cdot \pi^{1 / 2} \cdot \operatorname{erf}[\tau / 1000]}{500 \cdot \pi^{1 / 2} \cdot \operatorname{erf}[\tau / 1000]+\tau^{\prime}+\left[1-\exp \left(-(\tau / 1000)^{2}\right)\right] \cdot[4+(1+\zeta) \cdot 2]}
$$

It is worth noting that, although the binary parameter model can be graphically represented, it cannot find the optimal solution by using the analytic method when there are more than two parameters. Then, in the next section, we use PSO to perform multiparameters optimization in the time dimension.
4.4. Multiparameter Optimization. In this part, we suppose all parameters in equation (15) are unknown, except $I_{i}=1$. We consider the steady-state availability as a function of inspection interval. Let $\theta=1000, \beta=2$, and $\zeta=0.05$, and then substituting these parameters into equation (27), we can obtain the system availability function:

$$
A=\frac{500 \cdot \pi^{1 / 2} \cdot \operatorname{erf}[\tau / 1000]}{500 \cdot \pi^{1 / 2} \cdot \operatorname{erf}[\tau / 1000]+\tau^{\prime}+\left[1-\exp \left(-(\tau / 1000)^{2}\right)\right] \cdot[a+(1+\zeta) \cdot b]}
$$




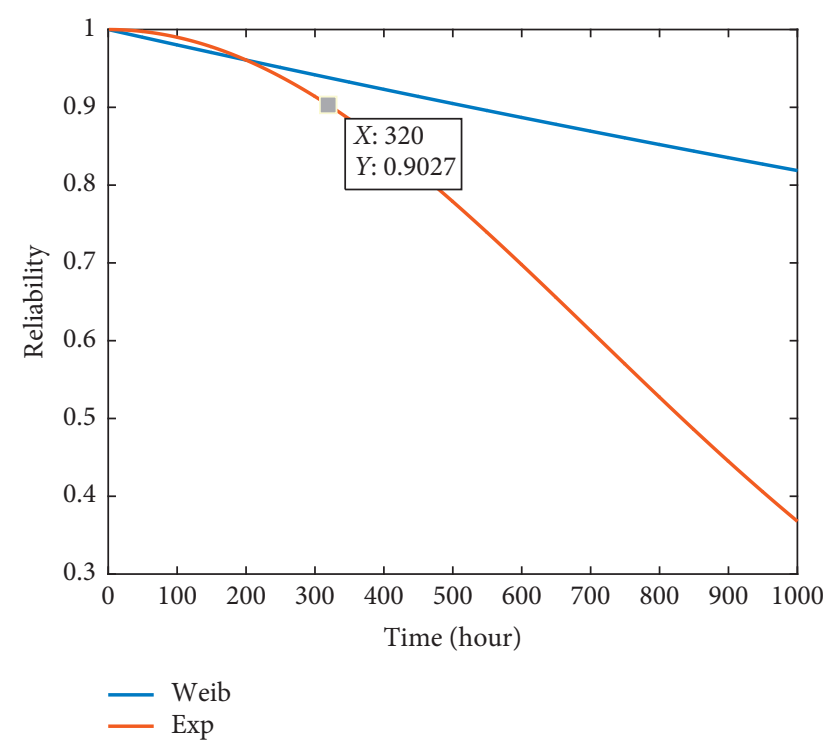

FIgURE 3: Single parameter optimization.

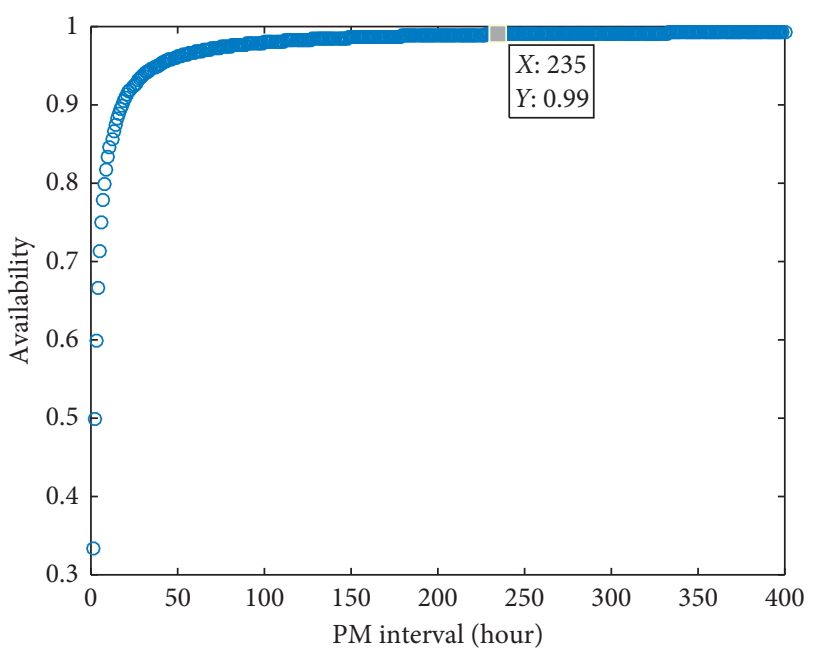

FIGURE 4: System reliability.

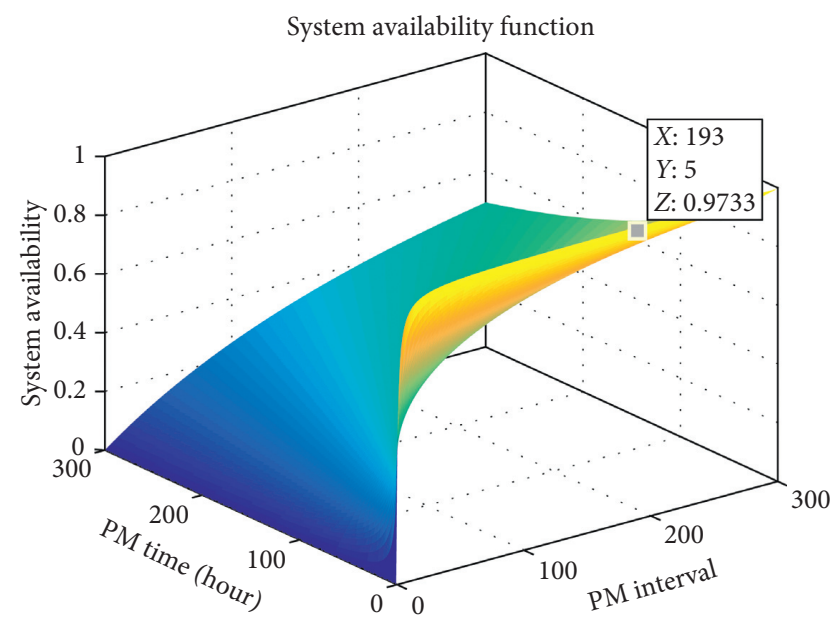

FIGURE 5: Binary parameter optimization PM interval and PM time.
TABle 1: Parameter selection.

\begin{tabular}{lc}
\hline Scholar & The value of $c_{1}$ and $c_{2}$ \\
\hline Clerc & $c_{1}=c_{2}=2.05$ \\
Carlisle & $c_{1}=2.8, c_{2}=1.3$ \\
Trelea & $w=0.6, c_{1}=c_{2}=1.7$ \\
Eberhart & $w=0.729, c_{1}=c_{2}=1.494$ \\
\hline
\end{tabular}

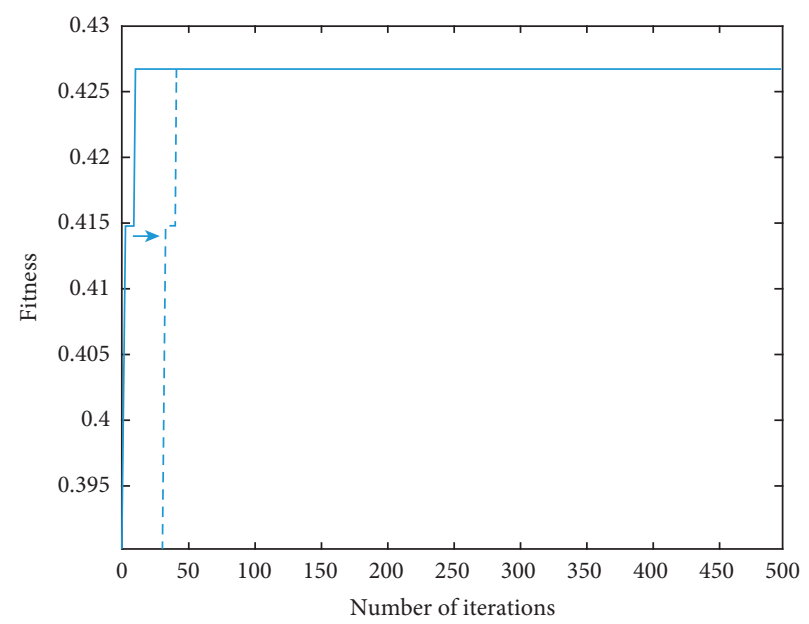

FIGURE 6: PSO evolution vs. number of iterations.

The parameters in particle swarm optimization have an important effect on the optimization performance. The parameter setting of the random number in the particle swarm optimization model is analyzed by the experiments [29-31]. The objective function is configured as equation (27), and the threshold range of the initial value of the particle position is $\tau=$ $x 1=320 * \operatorname{rand}(20,1) \sqrt{b^{2}-4 a c}, \tau^{\prime}=x 2=10 * \operatorname{rand}(20,1)$, $a=x 3=10 * \operatorname{rand}(20,1), b=x 4=10 * \operatorname{rand}(20,1)$, respectively.

Coefficients $c_{1}$ and $c_{2}$ have an important influence on the convergence of the algorithm during the calculation process. The literature [29] introduces a set of typical parameter setting methods, as shown in Table 1 .

Then, we choose the Carlisle value and utilize the MATLAB2016A to realize particle swarm optimization, and the result is shown in Figure $6(N=20, M=500, D=4)$.

As shown in Figure 6, the sets of parameters in algorithm are reasonable and the convergence speed is faster. The maximum system availability is 0.981383071167674 , and the parameters are, respectively, $\tau=292.2233489569377 /$ hour, $\tau^{\prime}=4.1597556475367 /$ hour, $a=9.3609676258832 /$ hour, and $b=5.3957053461250 /$ hour. At this time, the reliability is 0.918269829163875 , which meets the requirements of the system [28], and the results show that the design algorithm and model are correct.

Moreover, we calculate the proportional relationship of parameters: $T / \tau^{\prime} \approx 70.31, T / a \approx 31.22$ and $T / b=54.16$. It means that if system availability thresholds and reliability thresholds are given, thresholds for system mean repair time, test time, and delay time can be designed based on preventive maintenance interval.

Conversely, in the service stage, various general quality characteristics-related data can be collected to evaluate the 
availability of the system, so that the insufficiency in the system design stage is searched and suggestions for improvement can be proposed.

Although the threshold of each indicator in time dimension are obtained, if we want to further decompose into the design indicators of the system or subsystem, we need to obtain specific parameter models of various quality characteristics.

\section{Conclusion}

In this paper, the availability modelling and optimization of repairable systems with multigeneral quality characteristics are studied. Firstly, this paper derives the analytical results on the steady-state availability considering the reliability, maintainability, supportability, testability, and environmental parameters. Furthermore, the optimal method based on PSO is presented. A numerical example is presented to demonstrate the application of the developed approach algorithm. The methods and models presented in the paper can provide reference for the integrated design of equipment general quality characteristic index threshold.

As a preliminary analysis, obviously, there are numerous other extensions which are worth exploring. Firstly, although multiple general quality factors are considered, the research is far limited, many parameters are not considered, such as the fault detection rate and fault isolation rate in the testability, and the resource utilization rate in the supportability. Especially, the characterization method of environmental adaptability is too simple, which limits its application in engineering.

Additionally, the cost is also an important factor in optimizing availability, and if we can consider this factor, the engineering value is more stronger.

Finally, general quality characteristics will play more an important role in the design, service, and other life cycles of equipment. How to propose comprehensive measurement models with more extensive factors, more accurate method, and stronger applicability will be the future focus of research.

\section{Data Availability}

The data and conclusions used in the study are credible and open, which can be published and released by Mathematical Problems in Engineering. Further data can be obtained from the corresponding author upon request.

\section{Conflicts of Interest}

The authors declare that they have no conflicts of interest.

\section{Acknowledgments}

This study was co-supported by the NSF of China under Grant 51375490 and the China Postdoctoral Science Foundation (2019M653929).

\section{References}

[1] R. E. Barlow, F. Proschan, and L. C. Hunter, Mathematical Theory of Reliability, John Wiley \& Sons, New York, NY, USA, 1965.

[2] C. E. Ebeling, An Introduction to Reliability and Maintainability Engineering, Tsinghua University Press, Beijing, China, 2008.

[3] T. Yuo-Tern, W. Kuo Shong, and T. Hwei-Yuan, "Optimizing preventive maintenance for mechanical components using genetic algorithms," Reliability Engineering and System Safety, vol. 74, no. 1, pp. 89-97, 2001.

[4] T. Yuo-Tern, W. Kuo-Shong, and L.-C. Tsai, "A study of availability-centered preventive maintenance for multicomponent systems," Reliability Engineering and System Safety, vol. 84, no. 3, pp. 261-270, 2004.

[5] Y. Ibrahim, "Availability modelling and evaluation of a repairable system subject to minor deterioration under imperfect repairs," International Journal of Mathematics in Operational Research, vol. 7, no. 1, pp. 42-51, 2015.

[6] M. A. Hajeeh, "Availability of a system with different repair options," International Journal of Mathematics in Operational Research, vol. 4, no. 1, pp. 41-55, 2012.

[7] K. Ajay and K. G. Ramesh, "Availability analysis and evaluation of series parallel system using soft computing technique," International Journal of Reliability and Safety, vol. 10, no. 4, pp. 346-376, 2016.

[8] J. Sarkar and S. Sarkar, "Availability of a periodically inspected system under perfect repair," Journal of Statistical Planning and Inference, vol. 91, no. 1, pp. 77-90, 2000.

[9] J. Sarkar and S. Sarkar, "Availability of a periodically inspected system supported by a spare unit, under perfect repair or perfect upgrade," Statistics \& Probability Letters, vol. 53, no. 2, pp. 207-217, 2001.

[10] A. Biswas, J. Sarkar, and S. Sarkar, "Availability of a periodically inspected system, maintained under an imperfectrepair policy," IEEE Transactions on Reliability, vol. 52, no. 3, pp. 311-318, 2003.

[11] L. Cui and M. Xie, "Availability of a periodically inspected system with random repair or replacement times," Journal of Statistical Planning and Inference, vol. 131, no. 1, pp. 89-100, 2005.

[12] J. Li and K. Teng, "An availability calculation method for complex repairable systems," Acta Aeronauticaet Astronautica Sinica, vol. 38, no. 12, pp. 1-7, 2017.

[13] J. Li, Y. Chen, Y. Zhang, and H. Huang, "Availability modeling for periodically inspection system with different lifetime and repair-time distribution," Chinese Journal of Aeronautics, vol. 32, no. 7, pp. 1667-1672, 2019.

[14] T. Tang, D. Lin, D. Banjevic, and A. K. S. Jardine, “Availability of a system subject to hidden failure inspected at constant intervals with non-negligible downtime due to inspection and downtime due to repair/replacement," Journal of Statistical Planning and Inference, vol. 143, no. 1, pp. 176-185, 2013.

[15] L. Gao, X. Wu, and C. Chen, "Equpment availability and logistic delay probabitity," Journal of Academy of Force Engineering, vol. 19, no. 1, pp. 1-3, 2005.

[16] D.-H. Ding, "RMS integrated design and its demonstration," Electronic Product Reliability and Environmental Testing, vol. 32, no. 1, pp. 1-5, 2013.

[17] G. Kumar, V. Jain, and O. P. Gandhi, "Steady-state availability analysis of repairable mechanical systems with opportunistic maintenance by using Semi-Markov process," International 
Journal of System Assurance Engineering and Management, vol. 5, no. 4, pp. 664-678, 2014.

[18] M. E. Damcese and N. Temraz, "Analysis of availability and reliability of k-out-of-n: F model with fuzzy rates," International Journal of Computational Science and Engineering, vol. 10, no. 1/2, pp. 192-201, 2015.

[19] M. Ten Wolde and A. A. Ghobbar, "Optimizing inspection intervals-Reliability and availability in terms of a cost model: a case study on railway carriers," Reliability Engineering \& System Safety, vol. 114, pp. 137-147, 2013.

[20] Z. Simeu-Abazi and A. Alhouaij Ahmad, "Optimisation of distributed maintenance: modelling and application to the multi-factory production[J]," Reliability Engineering and System Safety, vol. 96, no. 11, pp. 1564-1575, 2011.

[21] Q. Qiu and L. Cui, "Reliability evaluation based on a dependent two-stage failure process with competing failures," Applied Mathematical Modelling, vol. 64, pp. 699-712, 2018.

[22] Q. Qiu, L. Cui, and H. Gao, "Availability and maintenance modelling for systems subject to multiple failure modes," Computers \& Industrial Engineering, vol. 108, pp. 192-198, 2017.

[23] Y. Li, Z. Yu, P. Rui, and X. Ma, "Opportunistic maintenance of production systems subject to random wait time and multiple control limits," Journal of Manufacturing Systems, vol. 47, pp. 12-34, 2018.

[24] Z.-L. Lin, Y.-S. Huang, and C.-C. Fang, "Non-periodic preventive maintenance with reliability thresholds for complex repairable systems," Reliability Engineering \& System Safety, vol. 136, no. 136, pp. 145-156, 2015.

[25] Y. Hajipour and S. Taghipour, "Non-periodic inspection optimization of multi-component and k-out-of-m systems," Reliability Engineering and System Safety, vol. 156, pp. 228243, 2016.

[26] Y. F. Yang, Y. W. Dai, and J. Feng, "Recession evolution rules and multi-objective decision-making of packaging machinery power machines considering environment factors," Packing Engineering, vol. 40, no. 3, pp. 186-192, 2019.

[27] J. H. Cao and K. Chen, Introduction to Reliability Mathematics, pp. 346-350, Higher education Press, Beijing, China, 2012.

[28] Chinese Military Standards (GJB), 1909A-2009: Demonstration of Reliability, Maintainability and Supportability Requirements for Materiel, Chinese Military Standards (GJB), Beijing, China, 2009.

[29] Z. X. Liu and H. Liang, "Parameter setting and experimental analysis of the random number in particle swarm optimization algorithm," Control Theory \& Applications, vol. 27, no. 11, pp. 1489-1496, 2010.

[30] C. M. N. A. Pereira, C. M. F. Lapa, A. C. A. Mol, and A. F. da Luz, "A Particle Swarm Optimization (PSO) approach for non-periodic preventive maintenance scheduling programming," Progress in Nuclear Energy, vol. 52, no. 8, pp. 710-714, 2010.

[31] C.-H. Wang and T.-W. Lin, "Improved particle swarm optimization to minimize periodic preventive maintenance cost for series-parallel systems," Expert Systems with Applications, vol. 38, no. 7, pp. 8963-8969, 2011. 